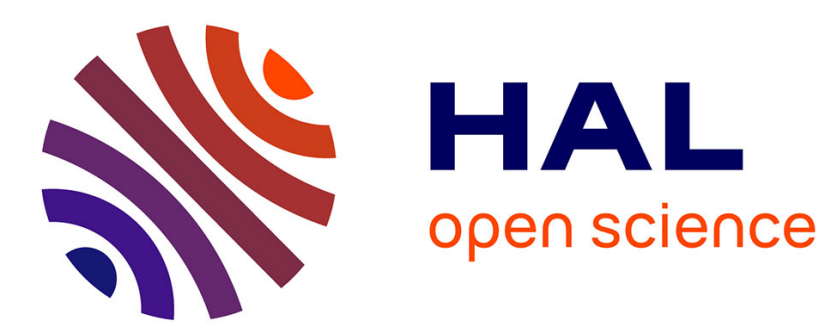

\title{
Monolithic generators of pseudo-nondiffracting optical vortex beams at the microscale
}

Albertas Žukauskas, Mangirdas Malinauskas, Etienne Brasselet

\section{To cite this version:}

Albertas Žukauskas, Mangirdas Malinauskas, Etienne Brasselet. Monolithic generators of pseudonondiffracting optical vortex beams at the microscale. Applied Physics Letters, 2013, 103 (18), pp.181122. 10.1063/1.4828662 . hal-00880445

\section{HAL Id: hal-00880445 \\ https://hal.science/hal-00880445}

Submitted on 13 Mar 2018

HAL is a multi-disciplinary open access archive for the deposit and dissemination of scientific research documents, whether they are published or not. The documents may come from teaching and research institutions in France or abroad, or from public or private research centers.
L'archive ouverte pluridisciplinaire HAL, est destinée au dépôt et à la diffusion de documents scientifiques de niveau recherche, publiés ou non, émanant des établissements d'enseignement et de recherche français ou étrangers, des laboratoires publics ou privés. 


\title{
Monolithic generators of pseudo-nondiffracting optical vortex beams at the microscale
}

\author{
Albertas Žukauskas, ${ }^{1}$ Mangirdas Malinauskas, ${ }^{1}$ and Etienne Brasselet ${ }^{2, a)}$ \\ ${ }^{1}$ Laser Nanophotonics Group, Department of Quantum Electronics, Physics Faculty, Vilnius University, \\ Sauletekio Ave. 9, LT-10222 Vilnius, Lithuania \\ ${ }^{2}$ Univ. Bordeaux and CNRS, Laboratoire Ondes et Matière d'Aquitaine, UMR 5798, F-33400 Talence, France
}

(Received 19 July 2013; accepted 18 October 2013; published online 1 November 2013)

\begin{abstract}
We report on the fabrication and characterization of micro-optical elements with typical size of $100 \mu \mathrm{m}$, which enable the production of pseudo-nondiffracting optical vortex beams of arbitrary order. This is made possible from the monolithic integration of spiral phase plates and axicons into helical axicons by direct laser writing using femtosecond laser nanopolymerization. The optical performances of the fabricated three-dimensional singular microstructures are experimentally measured and compared with their expected theoretical behavior, both in intensity and phase. The proposed approach thus represents an attempt to merge the field of singular integrated optics with that of nondiffracting light fields. (C) 2013 AIP Publishing LLC.
\end{abstract}

[http://dx.doi.org/10.1063/1.4828662]

Beam shaping of light using micron scale optical devices remains a contemporary challenge for the development of integrated optics, especially when the generation of optical singularities is considered. In fact, to imprint singular features onto a light beam from an element with typical spatial dimension $L$ basically requires an optical material endowed with an appropriate topological structuring having a spatial resolution $\delta L \ll L$. Consequently, the smaller is $L$, the more stringent is the constraint on $\delta L$. Still, nowadays, micro/nanofabrication techniques such as electron beam lithography, focused ion beam milling, and optical beam lithography enable arbitrary two- or three-dimensional material structuring with spatial resolution down to $\delta L \sim 10 \mathrm{~nm} .{ }^{1}$

In practice, microscopic high-resolution spiral phase plates delivering optical vortex beams have been realized, for instance, using electron beam lithography ${ }^{2}$ or femtosecond photopolymerization. ${ }^{3,4}$ Integrated optics ${ }^{5,6}$ and plasmonics ${ }^{7,8}$ strategies to generate vortex light fields have also emerged recently. Besides these high-tech solutions, an attractive alternative approach relies on light-induced spontaneous patterning of the optical anisotropy. By doing so, so called spin-orbit optical vortex generators can be realized at small scale, which has been shown by using amorphous glasses ${ }^{9}$ or liquid crystals. ${ }^{10,11}$ Finally, a purely natural strategy can also be considered by imprinting the topological information of liquid crystal topological defects to a light field that passes through it. ${ }^{12-14}$

However, the generation of vortex beams at the micron scale from usual Gaussian beams has been restricted so far to the case of Laguerre-Gauss-like diffracting fields. In contrast, here we consider the realization of micro-optical elements that enable the generation of high-order BesselGauss-like beams, hence pseudo-nondiffracting optical vortex beams whose zeroth-order nonvortex version has been originally introduced by Gori and coworkers. ${ }^{15}$ By doing so,

\footnotetext{
${ }^{\text {a)} E l e c t r o n i c ~ a d d r e s s: ~ e . b r a s s e l e t @ l o m a . u-b o r d e a u x 1 . f r ~}$
}

we aim at merging the field of singular integrated optics with that of nondiffracting light fields.

This is done by combining spiral phase plates, which transform Gaussian beams into Laguerre-Gaussian-like beams ${ }^{16}$ and axicons, which transforms Laguerre-Gaussian beams into high-order Bessel-like beams, ${ }^{17}$ into monolithic integrated optical elements. We thus obtain miniaturized versions of the so called helical axicons that have been realized so far at the macroscopic scale by using diffractive optical elements, namely, a $20 \mathrm{~mm} \times 20 \mathrm{~mm}$ device in Ref. 18 and a $4 \mathrm{~mm}$ diameter one in Ref. 19. This is done by direct laser writing using femtosecond laser nanopolymerization, ${ }^{20,21}$ benefiting from the previous developments regarding the realization of the micro-spiral phase plate ${ }^{22}$ and micro-axicon ${ }^{23}$ building blocks. The intensity and phase spatial features of the experimentally generated light fields are then quantitatively compared with theoretical predictions.

The proposed geometry of the monolithic micro-optical element is sketched in Fig. 1(a), where a three-dimensional structure made of an axicon and a spiral phase plate sharing a common basis is suspended on micropillars. The laser source used for fabrication is Yb:KGW ("PHAROS," Light Conversion Ltd.): 300 fs optical pulse duration, $200 \mathrm{kHz}$ repetition rate, second harmonic $(\lambda=515 \mathrm{~nm})$, under tight focusing conditions using a $63 \times, \mathrm{NA}=1.4$ microscope objective (Carl Zeiss). A complete description of the used system can be found elsewhere. ${ }^{24}$ The manufacturing of the monolithic component was performed as follows. (i) Fabrication of three support structures radially rotated at $120^{\circ}$ from each other, using $1 \mathrm{~mm} / \mathrm{s}$ scanning velocity and $110 \mu \mathrm{W}\left(0.603 \mathrm{TW} / \mathrm{cm}^{2}\right)$ laser irradiation. (ii) Spiral phase plates part were fabricated under $90 \mu \mathrm{W}\left(0.493 \mathrm{TW} / \mathrm{cm}^{2}\right)$ laser irradiation and using a reduced laser beam scanning velocity of $0.1 \mathrm{~mm} / \mathrm{s}$ to ensure the realization of a steep step, see Fig. 1(c). The scanning trajectory consists of concentric circles whose radii vary by $200 \mathrm{~nm}$ step in order to obtain a smooth surface. ${ }^{22}$ (iii) Axicons were produced under $110 \mu \mathrm{W}$ $\left(0.603 \mathrm{TW} / \mathrm{cm}^{2}\right)$ laser irradiation, using $1 \mathrm{~mm} / \mathrm{s}$ scanning 
(a)

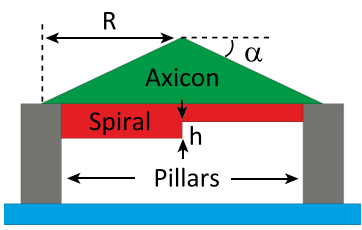

Substrate

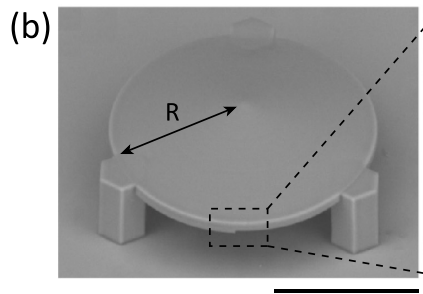

$50 \mu \mathrm{m}$
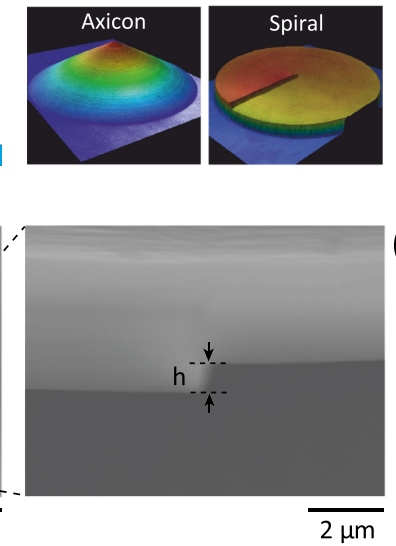

(c)

FIG. 1. (a) Left: side view of the proposed monolithic micro-optical generator of pseudo-nondiffracting optical vortex beams of order $\ell$ that consists of the combination of an axicon with a spiral phase plate made of the same material. Right: optical profilometry images of individual building blocks fabricated on the cover glass: an axicon with $\alpha=15^{\circ}$ and a spiral phase plate that corresponds to $\ell=1$ for $633 \mathrm{~nm}$ wavelength. Note that the different colors used for the spiral phase plate and the axicon merely emphasize the concept of merging two independent optical functionalities in a single monolithic element. (b) SEM image of a fabricated monolithic microstructure with $50 \mu \mathrm{m}$ radius, $\ell=1$ and $\alpha=15^{\circ}$. (c) Magnified view of the spiral step of the structure shown in panel (b).

velocity and spiral scanning trajectory with $100 \mathrm{~nm}$ voxel overlap. $^{23}$ The chosen material is a $\mathrm{Zr}$ containing hybrid organic-inorganic photopolymer ${ }^{25}$ photosensitized with $0.5 \%$ thioxanthen-9-one (Alfa Aesar). Samples were prepared by drop-casting on a glass substrate and pre-baking it for $30 \mathrm{~min}$ at $90^{\circ} \mathrm{C}$. After the exposure, photopolymer was immersed into pure 4-methyl-2-pentanone (Sigma Aldrich) developer for $1 \mathrm{~h}$ to remove the unexposed material, hence leaving free-standing microstructure on the substrate.

Basically, the helical axicon is described by two independent parameters: (i) the step height $h$ of the spiral plate and (ii) the inclination angle $\alpha$ of the axicon, see Fig. 1(a). These two parameters independently control the key features of the generated light field. On the one hand, $h$ is related to the topological charge $\ell$ of the generated on-axis optical phase singularity by the relationship $\ell=(n-1) h / \lambda$, where $n$ is the refractive index of the material and $\lambda$ is the wavelength. On the other hand, $\alpha$ is related to the opening angle $\theta_{\mathrm{B}}$ of the cone on which propagate the constitutive plane waves of an ideal nondiffracting Bessel beam ${ }^{26}$ following the relationship $\theta_{\mathrm{B}}=\arcsin [(n-1) \sin \alpha]-\alpha$ that simplifies to $\theta_{\mathrm{B}} \simeq(n-1) \alpha$ in the limit of small $\alpha$.

Two sets of microstructures have been fabricated: (i) $\ell=1$ with $\alpha=5^{\circ}, 10^{\circ}$, and $15^{\circ}$ and (ii) $\alpha=10^{\circ}$ with $\ell=1$, 2 , and 3 . In all cases, the monolithic structure is supported by three pillars with $30 \mu \mathrm{m}$ height and $20 \mu \mathrm{m}$ diameter, fixed to the substrate thus leaving enough space underneath to wash-out the unexposed photopolymer, and whose locations define an equilateral triangle. A scanning electron microscope (SEM) image of a $2 R=100 \mu \mathrm{m}$ diameter structure is shown in Fig. 1(b) in the case $\ell=1$ and $\alpha=15^{\circ}$, which qualitatively confirm the overall quality of the fabricated microoptical element. In addition, a SEM magnified view of the spiral phase plate step is shown in Fig. 1(c) from which we measure $h_{\mathrm{exp}} \simeq 1.29 \mu \mathrm{m}$. According to the refractive index determination of the polymerized material at $633 \mathrm{~nm}$ wavelength, $n=1.504,{ }^{22}$ the measured height differs from the expected value by $40 \mathrm{~nm}$. This corresponds to a $\sim 3 / \ell \%$ accuracy regarding the value of $\ell$. Also, within our experimental fabrication conditions, we obtain a surface roughness of $\sim 45 \mathrm{~nm}$. The quality of the surface is therefore better than $\lambda / 14$ for the wavelength used for optical characterization. That is to say, the obtained surfaces have a fairly good optical quality when compared with standard commercial macroscopic optical elements.

Optical performances of the fabricated devices are first tested regarding the propagation features of the generated light field. This is done by using a fundamental Gaussian probe beam obtained from a $\mathrm{He}-\mathrm{Ne}$ laser operating at $633 \mathrm{~nm}$. The probe beam is focused at normal incidence onto the sample with a divergence angle of $\theta_{\mathrm{G}} \sim 0.02 \mathrm{rad}$ that leads to a Gaussian beam waist $w_{0} \sim 10 \mu \mathrm{m}$ in the focal plane, where is placed the microscopic helical axicon. The intensity profiles at a distance $z$ from the midplane of the structure are recorded by means of a microscope objective $(40 \times, \mathrm{NA}=0.65)$ and a CCD camera.

The dependence of the azimuthally averaged intensity profiles as a function of $z$ is shown in Fig. 2(a) for $\ell=1$ and $\alpha=5^{\circ}$. In this figure, the radial intensity profiles are plotted in units of $r_{\max }$ where $r_{\max }$ is the radius of the first intensity maximum at $z=z_{\max }=w_{0} /[(n-1) \alpha]$. The distance $z_{\max }$ corresponds to the geometrical estimation of the distance over which a Bessel-Gauss beam generated by a Gaussian beam passing through an axicon has a pseudo-nondiffracting behavior $^{26}$ provided $z_{\max } / z_{0}>1$, where $z_{0}=\pi w_{0}^{2} / \lambda$ is the Rayleigh distance of the incident Gaussian beam.

In practice, the positioning of the sample with respect to the probe beam focal plane is not ensured with high accuracy. Therefore, consistency between experiments and simulations is ensured by using the incident Gaussian beam waist

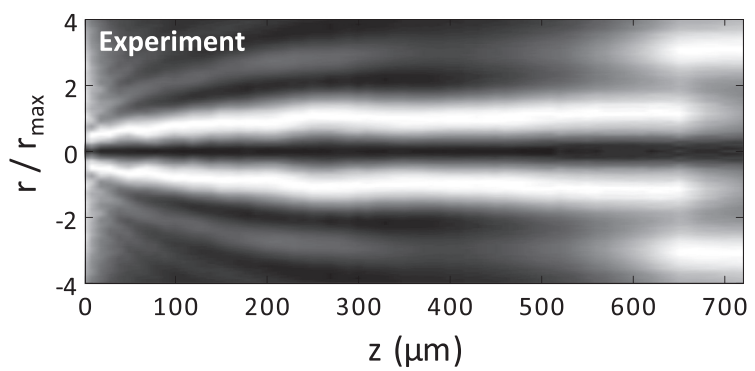

(a)

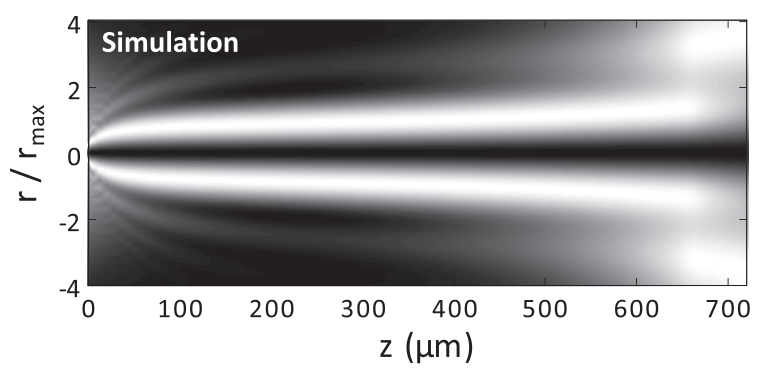

(b)

FIG. 2. Radial intensity profile as a function of the propagation distance $z$ for $\ell=1$ and $\alpha=5^{\circ}$. (a) Experimental data. (b) Simulations with $w_{0}=16.6 \mu \mathrm{m}$. For each $z$, the radial profiles are normalized to their maximal value. 


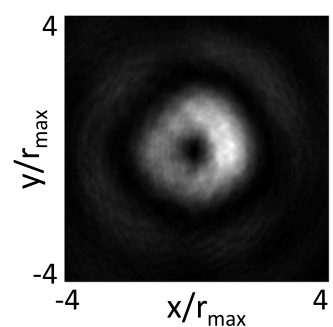

(a)

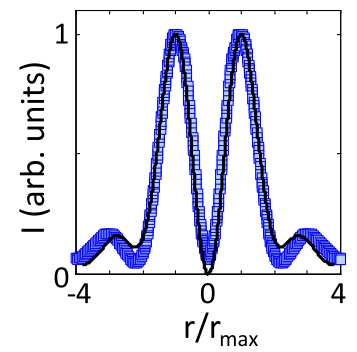

(b)

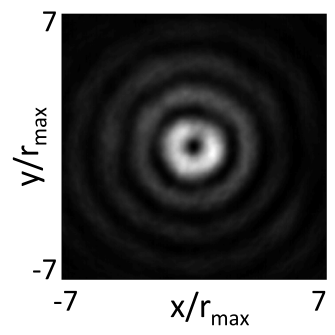

(c)

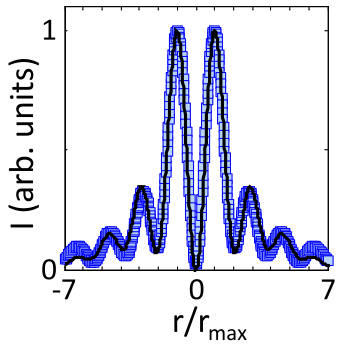

(d)

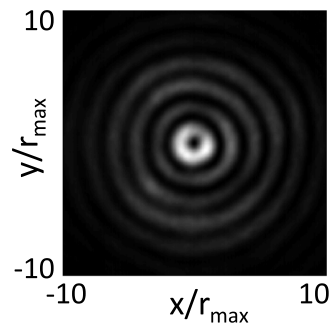

(e)

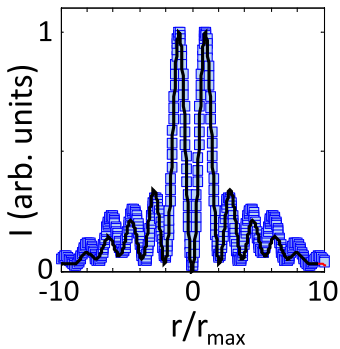

(f)

FIG. 3. Radial intensity profile as a function at $z=z_{\max }$ for $\ell=1$ and $\alpha=5^{\circ}$ ((a) and (b)), $\alpha=10^{\circ}$ ((c) and (d)), and $\alpha=15^{\circ}$ ((e) and (f)), for which $r_{\max }=3.6,1.9$, and $1.2 \mu \mathrm{m}$, respectively. Azimuthally averaged experimental data (square markers) are compared with simulations (solid curves) on the right column.

$w_{0}$ as the single adjusting parameter in the calculation. The simulations are carried out by considering the paraxial approximation of the Fresnel diffraction of a Gaussian beam by the phase mask of a helical axicon, discarding the residual contribution of the incident field outside the structure since $w_{0}$ is sufficiently smaller than $R(2-3$ times smaller is enough in practice $^{22}$ ). Namely,

$$
\begin{aligned}
I(r, z) \propto & \mid \int_{0}^{\infty} \exp \left[i k_{0} \rho^{2} /(2 z)-\rho^{2} / w_{0}^{2}\right] \exp \left[-i k_{0} \rho(n-1) \tan \alpha\right] \\
& \times\left. J_{\ell}(k r \rho / z) \rho d \rho\right|^{2}
\end{aligned}
$$

The optimization procedure eventually gives $w_{0}=16.6 \mu \mathrm{m}$ for the incident Gaussian beam waist value, hence $z_{\max }=380 \mu \mathrm{m}$, see Fig. 2(b).

The agreement regarding the propagating behavior of the light field emerging from the structure shown in Fig. 2 between the experiment data and simulations is fairly good. In particular, we observe the on-axis line of darkness that is characteristic of the presence of an optical vortex. Such a behavior does not depend on the angle $\alpha$ of the helical axicon. This is illustrated in Fig. 3, where the azimuth averaged radial intensity profiles is shown for $\ell=1$ with $\alpha=5^{\circ}, 10^{\circ}$, and $15^{\circ}$, which quantitatively match the simulations done from Eq. (1). The topological charge $\ell$ of the on-axis optical phase singularity can also be experimentally determined.
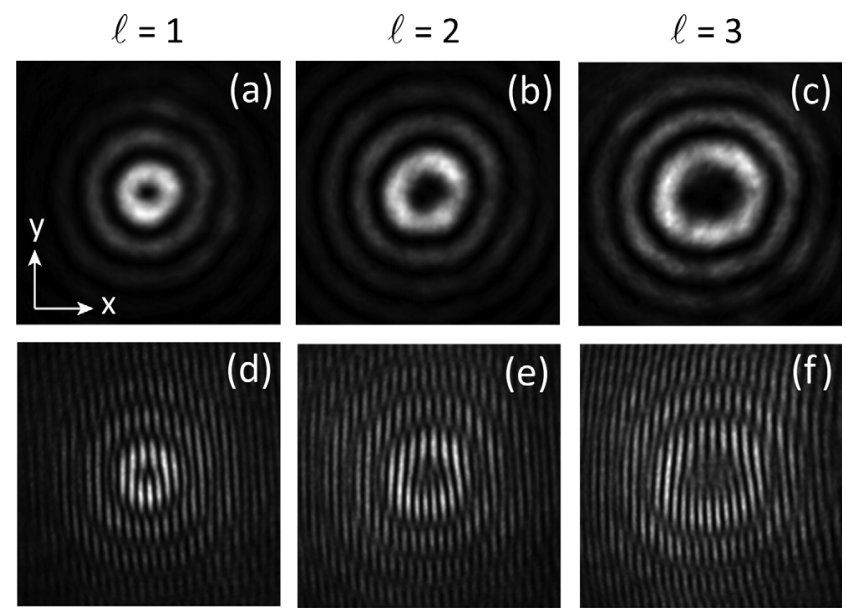

FIG. 4. Characterization of higher-order Bessel beams with topological charge $\ell=1$ ((a) and (b)), $\ell=2$ ((c) and (d)), and $\ell=3$ ((e) and (f)) for $\alpha=10^{\circ}$. Upper row: intensity profile. Bottom row: interference pattern exhibiting the typical fork pattern. All plots are shown on the same scale.

This is done by visual inspection of the interferogram with a reference Gaussian beam, as shown in Fig. 4 for $\ell=1, \ell=2$, and $\ell=3$ for $\alpha=10^{\circ}$. The characteristic "forked" intensity patterns associated with the $2 \ell \pi$ circulation of the optical phase around the beam axis are indeed clearly identified. Interestingly, we also notice an oscillating radial behavior of the field amplitude around zero, which is reminiscent of the Bessel feature of the generated light field. This can be grasped from the panels in the bottom part of Fig. 4 where an abrupt phase shift of $\pi$ for the field at radial distances that corresponds to null intensity values is easily identified.

Noteworthy, one should keep in mind that the generated high-order Bessel-Gauss beams only behave as pseudonondiffracting beams over the typical distance $z_{\max }$ if the condition $z_{\max } / z_{0}>1$ is satisfied, or equivalently, $\theta_{\mathrm{B}}<\theta_{\mathrm{G}}$. That is to say, when the Bessel nondiffracting features prevail over the Gaussian divergence characteristics. This imposes a constraint on the characteristic angle $\alpha$ of the axicon at given helical axicon radius $R$. Indeed, from previous practical finding that a complete transformation of a Gaussian beam into a Laguerre-Gaussian like beam by a spiral phase plate typically requires $w_{0} \leqslant R / 2,{ }^{22}$ one gets $\theta_{\mathrm{B}} \lesssim 2 \lambda /(\pi R)$ that gives $\alpha \lesssim 2 \lambda /[\pi(n-1) R]$. With present parameters $\lambda \simeq 0.6 \mu \mathrm{m}, n \simeq 1.5$, and $R=50 \mu \mathrm{m}$ we find the safe condition $\alpha \lesssim 1^{\circ}$ (obviously, if $w_{0}<R / 2$, the possible range for $\alpha$ increases). Although this is not the case for the presented structures with $\theta_{\mathrm{B}}>2.5^{\circ}$ for the used incident Gaussian beam divergence $\theta_{\mathrm{G}} \sim 1^{\circ}$, there is no lack of generality since arbitrarily small values of $\alpha$ can formally be achieved using femtosecond laser nanopolymerization.

To conclude, we have shown that femtosecond direct laser writing can be used to realize photopolymerized monolithic singular micro-optical elements for the generation of pseudo-nondiffracting high-order optical vortex beams. Singular beam shaping strategy at the micron scale could find applications in optical trapping and micromanipulation, where the concept of nondiffracting light optical fields has already been found useful. ${ }^{26}$ 
A.Ž. and M.M. are grateful for this research funding by a grant "MikroŠviesa" (No. MIP-12241) from the Research Council of Lithuania. E.B. was supported by EC's Seventh Framework Programme (LASERLAB-EUROPE, Grant Agreement No. 228334, OPTOBIO).

${ }^{1}$ Z. Gan, Y. Cao, R. A. Evans, and M. Gu, Nat. Commun. 4(1-7), 2061 (2013).

${ }^{2}$ W. C. Cheong, W. M. Lee, X.-C. Yuan, L.-S. Zhang, K. Dholakia, and H. Wang, Appl. Phys. Lett. 85, 5784 (2004).

${ }^{3}$ G. Knöner, S. Parkin, T. A. Nieminen, V. L. Y. Loke, N. R. Heckenberg, and H. Rubinsztein-Dunlop, Opt. Express 15(7), 5521-5530 (2007).

${ }^{4}$ X.-F. Lin, G.-Q. Hu, Q.-D. Chen, L.-G. Niu, Q.-S. Li, A. Ostendorf, and H.-B. Sun, Appl. Phys. Lett. 101, 113901 (2012).

${ }^{5}$ X. Cai, J. Wang, M. J. Strain, B. Johnson-Morris, J. Zhu, M. Sorel, J. L. O'Brien, M. G. Thompson, and S. Yu, Science 338, 363-366 (2012).

${ }^{6}$ S. A. Schulz, T. Machula, E. Karimi, and R. W. Boyd, Opt. Express 21, 16130 (2013).

${ }^{7}$ P. Zilio, E. Mari, G. Parisi, F. Tamburini, and F. Romanato, Opt. Lett. 37, 3234-3236 (2012).

${ }^{8}$ Y. Gorodetski, A. Drezet, C. Genet, and T. W. Ebbesen, Phys. Rev. Lett. 110, 203906 (2013).

${ }^{9}$ E. Brasselet, A. Royon, and L. Canioni, Appl. Phys. Lett. 100, 181901 (2012).

${ }^{10}$ E. Brasselet, Phys. Rev. A 82, 063836 (2010).

${ }^{11}$ B. Yang and E. Brasselet, J. Opt. 15, 044021 (2013).
${ }^{12}$ D. Voloschenko and O. D. Lavrentovich, Opt. Lett. 25, 317-319 (2000).

${ }^{13}$ E. Brasselet, N. Murazawa, H. Misawa, and S. Juodkazis, Phys. Rev. Lett. 103, 103903 (2009).

${ }^{14}$ C. Loussert, U. Delabre, and E. Brasselet, Phys. Rev. Lett. 111, 037802 (2013).

${ }^{15}$ F. Gori, G. Guattari, and C. Padovani, Opt. Commun. 64, 491-495 (1987).

${ }^{16}$ V. V. Kotlyar, A. A. Almazov, S. N. Khonina, V. A. Soifer, H. Elfstrom, and J. Turunen, J. Opt. Soc. Am. A 22, 849-861 (2005).

${ }^{17}$ J. Arlt and K. Dholakia, Opt. Commun. 177, 297-301 (2000).

${ }^{18}$ S. N. Khonina, V. V. Kotlyar, V. A. Soifer, M. V. Shinkaryev, and G. V. Uspleniev, Opt. Commun. 91, 158-162 (1992).

${ }^{19}$ V. V. Kotlyar, A. A. Kovalev, R. V. Skidanov, O. Y. Moiseev, and V. A. Soifer, J. Opt. Soc. Am. A 24, 1955-1964 (2007).

${ }^{20}$ H.-qB. Sun and S. Kawata, "Two-photon photopolymerization and 3D lithographic microfabrication," in NMR. 3D analysis. Photopolymerization (Springer, Berlin/Heidelberg, 2004).

${ }^{21}$ M. Farsari and B. Chichkov, Nat. Photon. 3, 450-452 (2009).

${ }^{22}$ E. Brasselet, M. Malinauskas, A. Žukauskas, and S. Juodkazis, Appl. Phys. Lett. 97, 211108 (2010).

${ }^{23}$ A. Žukauskas, M. Malinauskas, C. Reinhardt, B. Chichkov, and R. Gadonas, Appl. Opt. 51(21), 4995-5003 (2012).

${ }^{24}$ M. Malinauskas, G. Kirsanske, S. Rekstyte, T. Jonavicius, E. Kaziulionyte, L. Jonusauskas, A. Žukauskas, R. Gadonas, and A. Piskarskas, Lith. J. Phys. 52(4), 312-326 (2012).

${ }^{25}$ A. Ovsianikov, J. Viertl, B. Chichkov, M. Oubaha, B. MacCraith, I. Sakellari, A. Giakoumaki, D. Gray, M. Vamvakaki, M. Farsari, and C. Fotakis, ACS Nano 2(11), 2257-2262 (2008).

${ }^{26}$ D. McGloin and K. Dholakia, Contemp. Phys. 46, 15-28 (2005). 